\title{
miR-124 Inhibits Growth and Invasion of Gastric Cancer by Targeting ROCK1
}

\author{
Cong-Bing Hu, Qiao-Lin Li, Jian-Fei Hu, Qiang Zhang, Jian-Ping Xie, Li Deng*
}

\begin{abstract}
MicroRNAs (miRNAs) act as critical regulators of genes involved in many biological processes. Aberrant alteration of miRNAs have been found in many cancers, including gastric cancer (GC), but the molecular mechanisms are not well understood. Herein, we investigated the role of miR-124 in GC. We found that its expression was significantly reduced in both GC tissue samples and cell lines. Forced expression of miR-124 suppressed GC cell proliferation, migration, and invasion. Furthermore, the Rho-associated protein kinase (ROCK1) was identified as a direct target of miR-124 in GC cells. Finally, silencing of ROCK1 showed similar effects as miR-124 overexpression, while supplementation of ROCK1 remarkably restored the cell growth and invasion inhibited by miR-124. Together, our data demonstrate that miR-124 acts as a tumor suppressor by targeting ROCK1, and posit miR-124 as a novel strategy for GC treatment.
\end{abstract}

Keywords: Gastric cancer - miR-124 - ROCK1 - proliferation - migration - invasion

Asian Pac J Cancer Prev, 15 (16), 6543-6546

\section{Introduction}

Gastric cancer (GC) possesses the fourth common form of human malignant tumors and causes the second leading cancer-related death worldwide (Jemal et al., 2011). Approximately $700,000 \mathrm{GC}$ patients die annually worldwide (DeSantis et al., 2013). Despite great advance in the research on tumourigenesis and progression of GC, the molecular mechanisms are still poorly understood. Thus, it is urgent to study the underling molecular mechanism of GC.

MicroRNAs (miRNAs) are single-strand, small noncoding RNAs that play important roles in posttranscriptional repression (Lal et al., 2008). MiRNAs bind to the 3'-untranslated region (3'-UTR) of target mRNA and suppress translation or induce mRNA degradation (Wang et al., 2014). MiRNAs regulate a wide range of cellular processes, including proliferation, differentiation, apoptosis, and motility (Llaurado et al., 2014). Aberrant alteration of miRNA expression has been found in many cancers, and miRNAs act as oncogenes or tumorsuppressor genes (Hsu et al., 2014, Zhou et al., 2014). Increasing evidence reveals that miRNAs dysfunction was associated with multiple human cancers (Chu et al., 2014, Ding et al., 2014). In GC, several miRNAs were deregulated (Chen et al., 2013, Xia et al., 2014). MiR-124 was decreased in many cancers, and acted as a tumor suppressor (Li et al., 2013, Zhang et al., 2013). However its role in GC remains elusive.

Here we found that the expression of miR-124 was reduced in GC tissues and cell lines. Overexpression of miR-124 repressed the proliferation and metastasis of GC cells in vitro via targeting the Rho-associated protein kinase (ROCK1).

\section{Materials and Methods}

Human tissues, cell lines, and transfection

32 paired human GC samples and adjacent non-tumor tissues were obtained in our hospital. Written informed consent was obtained from all participants. This work was approved by the Ethics Committee of The First Affiliated Hospital, Yangtze University. Human GC cell lines, SGC-7901, BGC-823, and MKN-28, and normal gastric epithelial cell, GES-1, were all purchased from the the Chinese Academy of Sciences (Shanghai, China). Cells were cultured in RPMI 1640 medium, supplemented with $10 \% \mathrm{FBS}$ at $37{ }^{\circ} \mathrm{C}$ in an incubator containing $5 \% \mathrm{CO}_{2}$.

RNA isolation and quantitative real time PCR ( $q R T-P C R)$

Total RNAs were extracted with Trizol (Invitrogen, Carlsbad, CA, USA). MiRNAs were isolated with a miRNA Extraction Kit (Tiangen, Beijing, China). Then, the Poly (A) was added, and reversely transcribed into cDNA. The qRTPCR was performed using SYBR mix from Takara (Tokyo, Japan). The following primers were used: ROCK1 forward 5'-AAGAGAGTGATATTGAGCAGTTGCG-3', reverse 5'-TTCCTCTATTTGGTACAGAAAGCCA-3'; GAPDH forward 5'-GGTGGTCTCCTCTGACTTCAACA-3', reverse 5'-GTTGCTGTAGCCAAATTCGTTGT-3'. 
Cong-Bing Hu et al

Quantification was performed using the $2^{-\Delta \Delta \mathrm{Ct}}$ method. Primers of U6 and miR-124 were obtained from GeneCopoeia (MD, USA). U6 or GAPDH was used as endogenous control.

\section{Plasmids}

MiR-124 and the control mimics were obtained from RiboBio (Guangzhou, China). The 3'-UTR of ROCK1 which contains the potential binding sites of miR-124 was amplified using the following primer: forward 5'-CCCTCGAGTTTATTGCGGGTTTGC-3', reverse 5'-TTGCGGCCGCATTACTGAGGAGGTATTTGG-3'.

The PCR product was cloned into psiCHECK2 vector (Promega, Madison, WI, USA) within XhoI/NotI restriction sites. Mutation was performed with a mutation Kit (Stratagene, La Jolla, CA, USA). ROCK1 shRNA was purchased from Santa Cruz Biotechnology (Starr County, TX, USA). ROCK1 (NM_005406.2) was obtained from Origene (Rockville, MD, USA) and cloned into pcDNA3.0 vector (Invitrogen, Carlsbad, CA, USA).

\section{Cell viability assay}

The cell viability was determined by MTT assay (Sigma, St. Louis, MO, USA). $5 \times 10^{3}$ transfected cells were plated in 96-well plates. After incubation for different time, the culture medium was removed and MTT was added. After incubation for another $4 \mathrm{~h}$, the culture medium was replaced with DMSO, and OD570 was measured by a Microplate Reader (Molecular Deviced, Sunnyvale, CA, USA).

\section{Migration and invasion assays}

Cell migration and invasion assays were performed using transwell chamber. For migration, $2 \times 10^{4}$ transfected cells in serum-free medium were plated into the top chamber (BD, Bedford, MA, USA). For invasion, the same density of cells was seeded into the top chamber, which was pre-coated with Matrigel (BD, Bedford, MA, USA). After incubation for $24 \mathrm{~h}$, the membranes were fixed, stained with $0.1 \%$ crystal violet. Cells passing through the membranes were counted under microscope (Olympus, Tokyo, Japan).

\section{Luciferase activity assay}

$5 \times 10^{3}$ HED293 cells were plated in 96-well plates, and transfected with a mixture of $0.02 \mu \mathrm{g}$ psiCHECK2ROCK1 (wild type or mutant) and $100 \mathrm{nM}$ miR-124 mimic. After incubation for $24 \mathrm{~h}$, cells were collected for luciferase activity assays using the Dual-Luciferase Reporter Assay System (Promega, Madison, WI, USA). Renilla and firefly luciferase activity was normalized with Fly luciferase activity.2

\section{Western blotting}

Cells were harvested and lysed with lysis buffer (Beyotime, Shanghai, China). Proteins were separated by $10 \%$ SDS-PAGE, transferred to PVDF membranes. After blocking, membranes were immunoblotted with primary antibodies overnight at $4{ }^{\circ} \mathrm{C}$, followed by incubation with HRP-linked secondary antibodies for $1 \mathrm{~h}$. The signals were developed with ECL system (Millipore, Billerica,

WI, USA). Relative intensity of ROCK1 was normalized with GAPDH.

\section{Statistical analysis}

All data were expressed as mean \pm SD using the SPSS 16.0 software. The differences among the groups were estimated by one-way ANOVA or Student's t-test. $P<0.05$ was considered as statistically significant.

\section{Results}

miR-124 was reduced in GC

Expression of miR-124 in 32 pairs of GC tissues and the matched healthy tissues was measured using qRT-PCR. The expression of miR-124 was significantly reduced in GC tissues compared with the matched healthy tissues (Figure 1A). In addition, the expression of miR-124 was also significantly reduced in 3 GC cell lines, SGC-7901, BGC-823 and MKN-28, compared with the normal gastric epithelial cell, GES-1 (Figure 1B).

miR-124 inhibited growth and invasion of GC cells

To study the role of miR-124 in the regulation of growth and invasion of GC cells, we transfected miR124 or the control mimics into SGC-7901 cells (Figure 2A). MTT assay and migration and invasion assays were performed. MTT assay showed that miR-124
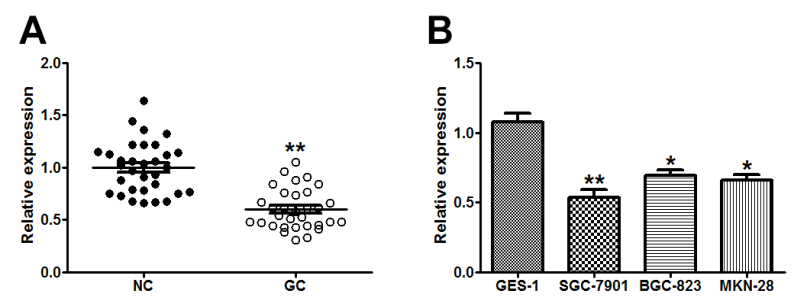

Figure 1. miR-124 was Reduced in GC. (A) miR-124 expression was determined by qRT-PCR in 32 paired GC tissues and matched healthy tissues. (B) The expression ofmiR-124 was analyzed in GC cell lines, SGC-7901, BGC-823 and MKN-28, and normal gastric epithelial cell, GES-1. ${ }^{*} p<0.05$, ${ }^{* *} p<0.01$ compared with control

A

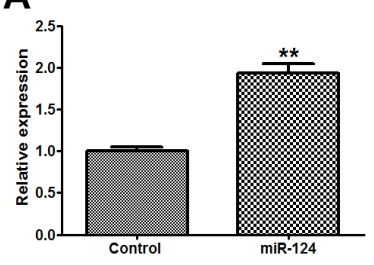

C

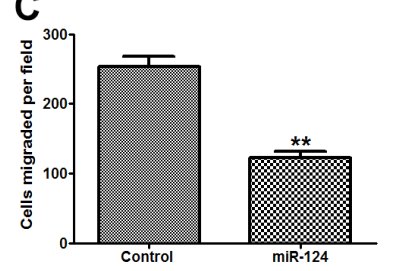

Figure 2. miR-124 Inhibited Growth and Invasion of

GC Cells. (A) SGC-7901 cells were transfected with miR-124 or the control mimics, and qRT-PCR was performed to detect the expression of miR-124. (B) MTT assay was performed to analyze the cell proliferation of SGC-7901 cells. (C, D) In vitro migration and invasion assays. Experiments were performed in triplicate. ${ }^{*} p<0.05, * * p<0.01$ compared with control 
A

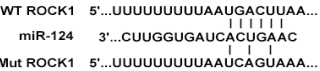

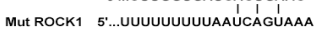

B

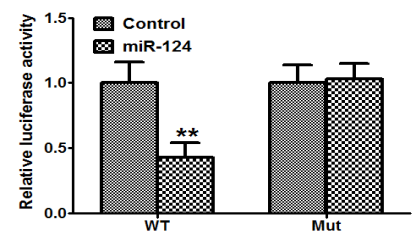

C

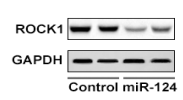

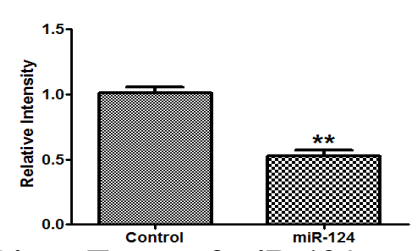

Figure 3. ROCK1 was a Direct Target of miR-124. (A) The 3'-UTR sequence of ROCK1 contains possible binding site for miR-124. (B) HEK293 cells were co-transfected with miR124 and wild type (WT) or mutant (Mut) 3'-UTR of ROCK1. After incubation for $24 \mathrm{~h}$, luciferase activity was assayed. (C) Protein level of ROCK1 was detected by Western blotting in SGC-7901 cells transfected with miR-124 or the control mimics. Experiments were performed in triplicate. ${ }^{*} p<0.05, * * p<0.01$ compared with control
A

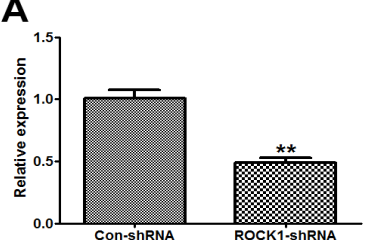

C

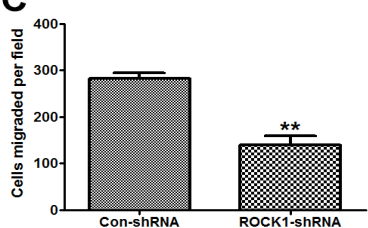

B

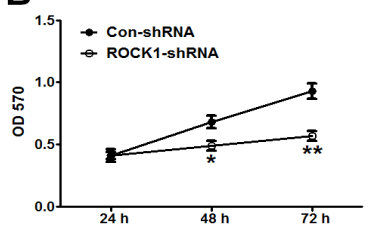

D

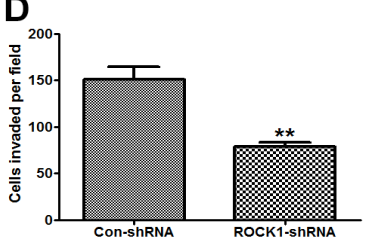

Figure 4. Silencing of ROCK1 Inhibited Ggrowth and Invasion of GC Cells. (A) SGC-7901 cells were transfected with ROCK1 shRNA or the control, and qRT-PCR was performed to detect the expression of ROCK1.(B) MTT, (C) migration, and (D) invasion of SGC-7901 cells transfected with ROCK1 shRNA or the control. Experiments were performed in triplicate. ${ }^{*} p<0.05,{ }^{*} p<0.01$ compared with control

overexpression remarkably reduced the proliferation of SGC-7901 cells (Figure 2B). Moreover, forced expression of miR-124 significantly suppressed both migration and invasion of SGC-7901 cells (Figure 2C, D).

\section{ROCK1 was a direct target of $m i R-124$}

To identify target of miR-124, TargetScan 6.2 was used. ROCK1 contains potential binding sites of miR-124 (Figure 3A). Luciferase activity assay found that miR-124 significantly reduced the luciferase activity of the wild type (WT) but not the mutant (Mut) 3'-UTR of ROCK1 in HEK293 cells (Figure 3B). Moreover, Overexpression of miR-124 significantly suppressed ROCK 1 protein levels (Figure 3C). These data suggest that ROCK1 was a direct target of miR-124.

Silencing of ROCK1 inhibited growth and invasion of GC cells

We further studied whether silencing of ROCK1
A

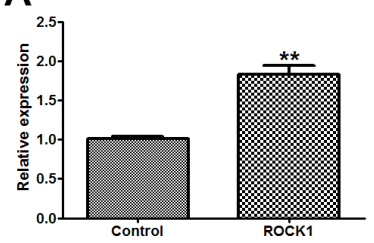

C

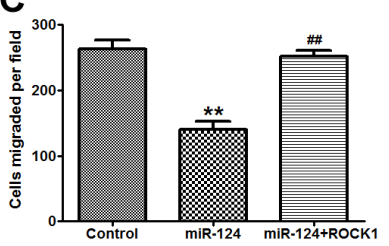

B

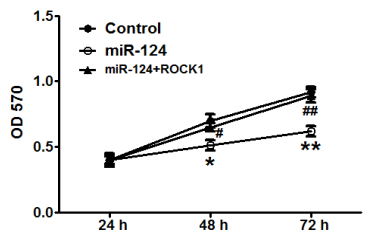

D

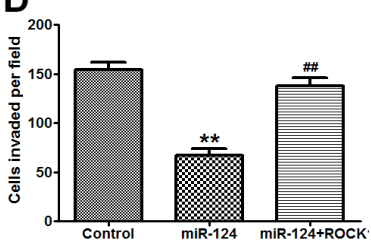

Figure 5. Supplement of ROCK1 Attenuated the Effects of miR-124. (A) SGC-7901 cells were transfected with pcDNA-ROCK 1 or the vector, and the expression of ROCK1 was determined by qRT-PCR. (B) SGC-7901 cells were co-transfected with miR-124 and pcDNA-ROCK1 or the vector, and MTT assay was performed. (C) Migration and (D) Invasion assays. Experiments were performed in triplicate. ${ }^{*} p<0.05,{ }^{*} \mathrm{P}<0.01$ compared with control. $\# p<0.05, \# \# p<0.01$ compared with miR-124 group

performed similar effects with miR-124 overexpression. ROCK1 shRNA or the control were transfected into SGC7901 cells, and the ROCK1 mRNA levels was determined by qRT-PCR (Figure 4A). We found that silencing of ROCK1 significantly suppressed cell proliferation, migration and invasion (Figure 4B-D), similar with the effects of miR-124 overexpression.

Supplement of ROCK1 attenuated the effects of miR-124

We further investigated whether restoration of ROCK1 could reverse the tumor suppressive effect of miR-124. MTT, migration and invasion assays (Figure $5 \mathrm{~A}-\mathrm{C}$ ) showed that restoration of ROCK1 significantly reversed the tumor suppressive effect of miR-124 on SGC-7901 cells. The efficience of pcDNA3-ROCK1 was determined by qRT-PCR (Figure 5D). These data suggest that supplement of ROCK1 significantly attenuated tumor suppressive effect of miR-124.

\section{Discussion}

Although many miRNAs had been aberrantly altered in GC, their underlying molecular mechanisms in GC development and progression still remain poorly understood (Hong et al., 2014). Thus exploring the function of miRNAs specifically involved in GC development and progression would greatly help expand our knowledge on $\mathrm{GC}$, and provide new targets for its diagnosis and therapy. In this study, we found that miR-124 was reduced in both GC tissues and cell lines. Ectopic expression of miR-124 reduced proliferation and motility of GC cells.

MiR-124 is a brain-enriched miRNA, which was significantly decreased in a variety of human malignances, including colorectal cancer, glioblastoma, hepatocellular carcinoma, medulloblastoma, and GC (Li et al., 2009, Xie et al., 2014). Zhang $\mathrm{Y}$ et al. reported that miR-124 could increase the radiosensitivity of colorectal cancer cells by blocking the expression of paired related homeobox 
1 (PRRX1) (Zhang et al., 2014). Lv Z et al. revealed that miR-124 inhibited proliferation of glioblastoma by targeting Son of sevenless homolog 1 (SOS1) (Lv and Yang 2013). Zhao WH et al. found that downregulation of miR-124 promoted the malignant progression of glioblastoma cells via increasing novel protein similar to human protein phosphatase 1 , regulatory (inhibitor) subunit 13 like (PPP1R3L) (Zhao et al., 2013). In GC, Xie L et al. reported that miR-124 inhibited proliferation and induced apoptosis by targeting enhancer of zeste homolog 2 (EZH2) (Xie et al., 2014). Xia J et al. found that miR-124 suppressed the proliferation of GC cell via targeting sphingosine kinase type 1 (SphK1) (Xia et al., 2012). In this study, we found that ROCK1 was direct target of miR-124 in GC cells, and miR-124 inhibited the proliferation and motility partially by targeting ROCK 1 . Our work further expanded the role of miR-124 in GC.

ROCK1 is a member of the Rho-associated serine/ threonine kinase family, which facilitates the reorganization of the actin cytoskeleton during motion (Wen et al., 2014). ROCK 1 has been found to be increased in many cancers, including glioma, osteosarcoma, prostate cancer, and GC (Zhang et al., 2013,Zhou et al., 2013). ROCK1 functioned as an oncogene, and possessed a wide range of functions, including migration, invasion, and metastasis (Shin et al., 2014). ROCK1 was targeted by several miRNAs, including miR-135a, miR-145, and miR-148a (Li et al., 2013, Shin et al., 2014, Wan et al., 2014). In gastric cancer, ROCK1 was positively correlated with TNM stage and LN metastasis (Wu et al., 2013). In the present study, we found that ROCK1 was a direct target of miR-124 in GC cells. Silencing of ROCK1 by shRNA inhibited proliferation, migration, and invasion of GC cell line, SGC-7901. Restoration of ROCK 1 in GC cells significantly reversed the tumor suppressive role of miR-124.

In conclusion, we have demonstrated that miR-124 suppressed gastric carcinogenesis by targeting ROCK1, suggesting that miR-124 might be a novel biomarker and therapeutic target for GC treatment.

\section{References}

Chen G, Shen ZL, Wang L, et al (2013). Hsa-miR-181a-5p expression and effects on cell proliferation in gastric cancer. Asian Pac J Cancer Prev, 14, 3871-5.

Chu D, Zhao Z, Li Y, et al (2014). Increased microRNA-630 expression in gastric cancer is associated with poor overall survival. PLoS One, 9, 90526.

DeSantis C, D Naishadham, A Jemal (2013). Cancer statistics for African Americans, 2013. CA Cancer J Clin, 63, 151-66.

Ding H, Wu YL, Wang YX, Zhu FF (2014). Characterization of the microRNA expression profile of cervical squamous cell carcinoma metastases. Asian Pac J Cancer Prev, 15, 1675-9.

Hong L, Han Y, Yang J, et al (2014). MicroRNAs in gastrointestinal cancer: prognostic significance and potential role in chemoresistance. Expert Opin Biol Ther, 14, 1103-11.

Hsu TI, Hsu CH, Lee KH, et al (2014). MicroRNA-18a is elevated in prostate cancer and promotes tumorigenesis through suppressing STK4 in vitro and in vivo. Oncogenesis, $\mathbf{3}, 99$.

Jemal A, Bray F, Center MM, et al (2011). Global cancer statistics. CA Cancer J Clin, 61, 69-90.
Lal A, Kim HH, Abdelmohsen K, et al (2008). p16(INK4a) translation suppressed by miR-24. PLoS One, 3, 1864.

Li J, Song Y, Wang Y, Luo J, Yu W (2013). MicroRNA-148a suppresses epithelial-to-mesenchymal transition by targeting ROCK1 in non-small cell lung cancer cells. Mol Cell Biochem, 380, 277-82.

Li KK, Pang JC, Ching AK, et al (2009). miR-124 is frequently down-regulated in medulloblastoma and is a negative regulator of SLC16A1. Hum Pathol, 40, 1234-43.

Li L, Luo J, Wang B, et al (2013). Microrna-124 targets flotillin-1 to regulate proliferation and migration in breast cancer. $\mathrm{Mol}$ Cancer, 12, 163.

Llauradó M, Majem B, Altadill T, et al (2014). MicroRNAs as prognostic markers in ovarian cancer. Mol Cell Endocrinol, 390, 73-84.

Lv Z, Yang L (2013). MiR-124 inhibits the growth of glioblastoma through the downregulation of SOS1. Mol Med Rep, 8, 345-9.

Shin JY, Kim YI, Cho SJ, et al (2014). MicroRNA 135a suppresses lymph node metastasis through down-regulation of ROCK1 in early gastric cancer. PLoS One, 9, 85205.

Wan X, Cheng Q, Peng R, et al (2014). ROCK1, a novel target of miR-145, promotes glioma cell invasion. Mol Med Rep, 9, 1877-82.

Wang X, Yu H, Lu X, et al (2014). MiR-22 suppresses the proliferation and invasion of gastric cancer cells by inhibiting CD151. Biochem Biophys Res Commun, 445, 175-9.

Wen X, Ding L, Wang JJ, et al (2014). ROCK1 and LIM Kinase Modulate Retrovirus Particle Release and Cell-Cell Transmission Events. J Virol, 88, 6906-21.

Wu YJ, Tang Y, Li ZF, et al (2013). Expression and significance of Rac1, Pak1 and Rock1 in gastric carcinoma. Asia Pac J Clin Oncol, 10, 33-9.

Xia J, Guo X, Yan J, Deng K (2014). The role of miR-148a in gastric cancer. J Cancer Res Clin Oncol. [Epub ahead of print]

Xia J, Wu Z, Yu C, et al (2012). miR-124 inhibits cell proliferation in gastric cancer through down-regulation of SPHK1. J Pathol, 227, 470-80.

Xie L, Zhang Z, Tan Z, et al (2014). microRNA-124 inhibits proliferation and induces apoptosis by directly repressing EZH2 in gastric cancer. Mol Cell Biochem. [Epub ahead of print]

Zhang C, Zhang S, Zhang Z, et al (2013). ROCK has a crucial role in regulating prostate tumor growth through interaction with c-Myc. Oncogene. [Epub ahead of print]

Zhang H, Wang Q, Zhao Q, Di W (2013). MiR-124 inhibits the migration and invasion of ovarian cancer cells by targeting SphK1. J Ovarian Res, 6, 84.

Zhang Y,Zheng L, Huang J, et al (2014). MiR-124 Radiosensitizes Human Colorectal Cancer Cells by Targeting PRRX1. PLoS One, 9, 93917.

Zhao WH, Wu SQ, Zhang YD (2013). Downregulation of miR124 promotes the growth and invasiveness of glioblastoma cells involving upregulation of PPP1R13L. Int J Mol Med, 32, 101-7.

Zhou P, Huang G, Zhao Y, et al (2014). MicroRNA-363-mediated downregulation of S1PR1 suppresses the proliferation of hepatocellular carcinoma cells. Cell Signal, 26, 1347-54.

Zhou X, Wei M, Wang W (2013). MicroRNA-340 suppresses osteosarcoma tumor growth and metastasis by directly targeting ROCK1. Biochem Biophys Res Commun, 437, 653-8. 